\title{
artigo
}

Santos, E.S.; Filgueiras, T.F.; Carvalho, M.A.; Mangueira, F.F.A.; Xavier, B.L.Q.; Soares, A.;

Conhecimento de enfermeiros acerca da diabetes mellitus gestacional

\section{Conhecimento de enfermeiros acerca da diabetes mellitus gestacional}

\author{
Knowledge of nurses about diabetes mellitus gestacional \\ Conocimiento de enfermeras sobre diabetes mellitus gestacional
}

\begin{abstract}
RESUMO
Relatar o conhecimento de enfermeiros acerca da diabetes mellitus gestacional. Trata-se de uma pesquisa de campo descritiva com abordagem qualitativa realizado com enfermeiros cadastrados na estratégia de saúde da família em um distrito sanitário de cidade paraibana. A coleta de dados foi realizada nos meses de setembro a outubro de 2019 com 18 enfermeiros. Os dados foram organizados por categorias temáticas resultantes da junção de todas as falas coletadas e separadas por unidade de sentido. As categorias temáticas apresentadas na discussão foram "identificação da diabetes mellitus gestacional"; "acompanhamento x encaminhamento"; "comportamentos de risco"; "muitas não podem seguir porque não tem condições financeiras". 0 entendimento dos enfermeiros sobre as consequências ocasionadas pelo diabetes gestacional é significante, pois interfere na qualidade da assistência ofertada. Foi evidente a necessidade de cuidado diferenciado a gestante com base no fator sócio situacional.
\end{abstract}

DESCRITORES: Diabetes gestacional; Enfermeiro; Atenção Básica.

\section{ABSTRACT}

Reporting nurses' knowledge about gestational diabetes mellitus. This is a descriptive field research with a qualitative approach carried out with nurses registered in the family health strategy in a health district of a city in Paraiba. Data collection was carried out from September to October 2019 with 18 (eighteen) nurses. The data were organized into thematic categories resulting from the combination of all the statements collected and separated by unit of meaning. The thematic categories presented in the discussion were "identification of gestational diabetes mellitus"; "Follow-up x referral"; "risk behaviors"; "Many women cannot follow because they have no financial conditions". The nurses' understanding of the consequences caused by gestational diabetes is significant, as it interferes with the quality of care offered. The need for differentiated care for pregnant women was evident based on the socio-situational factor.

DESCRIPTORS: Gestational diabetes; Nurse; Basic Attention.

\section{RESUMEN}

Informar sobre el conocimiento de las enfermeras sobre la diabetes mellitus gestacional. Esta es una investigación de campo descriptiva con un enfoque cualitativo realizada con enfermeras registradas en la estrategia de salud familiar en el distrito de salud III de la ciudad de João Pessoa-PB. La recopilación de datos se realizó de septiembre a octubre de 2019 con 18 enfermeras. Los datos se organizaron en categorías temáticas resultantes de la combinación de todas las declaraciones recopiladas y separadas por unidad de significado. Las categorías temáticas presentadas en la discusión fueron "identificación de diabetes mellitus gestacional"; "Seguimiento x referencia"; "comportamientos de riesgo"; "Muchos no pueden seguir porque no tienen condiciones financieras". La comprensión de las enfermeras de las consecuencias causadas por la diabetes gestacional es significativa, ya que interfiere con la calidad de la atención ofrecida. La necesidad de atención diferenciada para las mujeres embarazadas fue evidente en función del factor socio-situacional.

DESCRIPTORES: Diabetes gestacional; Enfermera; Atención primaria.

RECEBIDO EM: 28/07/2020 APROVADO EM: 31/07/2020

\section{Elaine da Silva Santos}

Enfermeira pelo Centro Universitário de João Pessoa.

ORCID: 0000-0002-7986-6231 
Thaynara Ferreira Filgueiras

Enfermeira pela UFCG. Mestra em Enfermagem pelo PPGENF/UFPB.

ORCID: 0000-0001-7520-4145

\section{Michele Alves de Carvalho}

Enfermeira pela UFPB. Mestra em Enfermagem pelo PPGENF/UFPB

ORCID: 0000-0002-5512-5041

\section{Francisco Fernandes Abel Mangueira}

Enfermeiro pela UFCG. Mestre em Saúde Pública pelo PPGSP/UEPB.

ORCID: 0000-0002-6111-7152

\section{Bárbara Letícia de Queiroz Xavier}

Enfermeira pela UFCG. Especialista em Saúde da Família pela UNILAB.

ORCID: 0000-0003-1622-9128

\section{Amanda Soares}

Enfermeira pela UFCG. Mestra em Saúde Pública pelo PPGSP/UEPB.

ORCID: 0000-0001-8063-4131

\section{INTRODUÇÃO}

0 diabetes é uma doença complexa e crônica que dispensa cuidados contínuos para além do controle glicêmico. Em mulheres, o principal fator de risco para o desenvolvimento de diabetes do tipo 2 e de síndrome metabólica é o antecedente obstétrico de Diabetes Mellitus Gestacional (1). O diabetes mellitus gestacional define-se como um subtipo de hiperglicemia diagnosticada ou detectada pela primeira vez no decurso da gravidez com níveis glicêmicos sanguíneos que não atingem os critérios diagnósticos para diabetes mellitus ${ }^{(2)}$.

Segundo os critérios utilizados pela Organização Mundial da Saúde, a prevalência de diabetes gestacional em mulheres acima de 20 anos atendidas no Sistema Único de Saúde (SUS) é de 7,6\% e cerca de 7\% de todas as gestações estão associadas a esta complicação, resultando em mais de 200.000 casos/ano. Em relação aos casos, $94 \%$ apresentam intolerância diminuída à glicose e, apenas $6 \%$ deles, atingem os critérios diagnósticos para o diabetes não gestacional ${ }^{(3)}$.

O diagnóstico universal da Organização Mundial da Saúde considera como fatores de risco para a diabetes a idade, sobrepeso/obesidade, antecedentes familiares de diabetes mellitus (primeiro grau), antecedentes pessoais de alterações metabólicas e obstétricos (4). No Brasil, para o rastreamento e diagnóstico de diabetes mellitus gestacional é o con- siderado o critério da viabilidade financeira e disponibilidade técnica total. Na presença destes, deve-se realizar a glicemia de jejum até 20 semanas de idade gestacional. Caso a glicemia de jejum apresente valores inferiores a $92 \mathrm{mg} / \mathrm{dL}$, deve-se realizar o teste oral de tolerância a glicose. Em situações de viabilidade financeira e disponibilidade técnica parcial o teste de tolerância deve ser substituído pela glicemia de jejum ${ }^{(5)}$.

A hiperglicemia durante a gestação e puerpério constitui um relevante problema da atualidade, em detrimento do risco de alteraçôes perinatais e de desenvolvimento de doenças a longo prazo para a mulher e criança. O aumento de sua prevalência é associado principalmente em decorrência da epidemia de obesidade em diversos países ${ }^{(6)}$.

No tocante às consequências da diabetes mellitus gestacional para o feto, uma das principais é a obesidade na primeira infância ${ }^{(7)}$. O objetivo do acompanhamento pré-natal é assegurar o desenvolvimento da gestação, com um parto de recémnascido saudável, sem impacto para a saúde materna ${ }^{(6)}$.

A estratégia de saúde da família é a porta de entrada preferencial da gestante no SUS. Os profissionais de saúde envolvidos no cuidado à gestante devem realizar ações de atenção integral, contemplando a proteção e a promoção da saúde, prevenção de agravos e escuta qualificada das necessidades das gestantes, proporcionando atendimento humanizado e estabelecimento de vínculo ${ }^{(8)}$.
Diante da problemática discutida, é inegável a importância do conhecimento do enfermeiro acerca das ações prestadas as gestantes com diabetes mellitus gestacional, tendo em vista as necessidades específicas de cada caso. Dessa forma, a intervenção prestada na atenção básica é essencial para o controle e/ou prevenção de complicações materno-fetal.

Para tanto, este estudo objetiva relatar o conhecimento de enfermeiros acerca da assistência de enfermagem à mulher com diabetes mellitus gestacional.

\section{MÉTODOS}

Esta pesquisa trata-se de um estudo de campo descritivo com abordagem qualitativa, foi realizada em unidades básicas de saúde da família do distrito sanitário III do município de João Pessoa no Estado da Paraíba.

A amostra não-probabilística foi composta por 18 enfermeiros delimitada com a inclusão daqueles que realizavam o acompanhamento de gestantes no serviço de saúde; e que possuíssem no mínimo 1 (um) ano de experiência na unidade de saúde da família. Já os critérios de exclusão que foram utilizados: enfermeiros que não estiveram presentes na unidade de saúde da família no momento da coleta de dados e não se enquadrar aos critérios de inclusão.

Os dados foram coletados nos meses de setembro a outubro de 2019, através de um questionário não validado. Com a conclu- 
são da coleta, os dados foram organizados por categorias temáticas resultantes da junção de todas as falas coletadas, embaralhando-as e fazendo a conexão das respostas que correspondam a uma unidade de sentido, apresentando temática semelhante independente do objetivo. A análise dos dados foi realizada de acordo com a análise de conteúdo temática definida por Minayo ${ }^{(9)}$.

$\mathrm{O}$ presente estudo foi aprovado pelo Comitê de Ética e Pesquisa do Centro Universitário de João Pessoa com o número de CAAE:16381319.0.0000.5176.

\section{RESULTADOS E DISCUSSÃO: \\ Caracterização da amostra}

Sobre as variáveis investigadas, observou-se que 77,8\% ( $n=14)$ dos enfermeiros que participaram da entrevista possuíam idade entre 30 a 50 anos. Quanto ao tempo de formação, 55,6\% $(\mathrm{n}=10)$ possuíam entre 21 a 37 anos; $94,4 \%(\mathrm{n}=17)$ atuavam na atenção primária a saúde entre quatro a vinte anos. A titulação acadêmica predominante no grupo foi a de enfermeiro sanitaristas com representação de $66,7 \%(\mathrm{n}=12)$.

\section{Categoria 01 - Identificação da Dia- betes Mellitus Gestacional}

Nessa categoria, foi possível identificar através das falas que o diagnóstico do diabetes gestacional é baseado, principalmente, nas alterações glicêmicas vigentes nos exames de rotina.

[...] é o aumento da glicemia da mulher durante a gestação. Ela ocorre quando a um desregulamento no metabolismo da gestante que já tem uma predisposição a desenvolver o diabetes gestacional. [...] (E10)

[...] é uma doença metabólica, que é causada pelo aumento de glicemia. Podemos definir como intolerância a glicose e açúcar excedido no sangue não parar de formar conteúdo para as células do sangue. [...] (E12)

As alteraçóes glicêmicas se relacionam com a fisiologia da doença. A diabetes gestacional acarreta consequências materno-infantil, sendo o principal fator de risco para o aumento da morbimortalidade infantil ${ }^{(10)}$. Baseado na necessidade de assistir e intervir a gestante com diabetes gestacional precocemente, justifica-se o conhecimento apresentado pelos enfermeiros deste estudo, mediante inserção e execução de políticas públicas de saúde destinadas a assistência materno-infantil no Brasil.

Destaca-se que a solicitação dos exames para verificação da glicemia foi citada como o padrão ouro no diagnóstico de diabetes gestacional. Conforme observa-se nos relatos dos enfermeiros:

[...] Durante as consultas de pré-natal solicitamos os exames de glicemia de jejum, curva glicêmica e o teste de intolerância a glicose para a identificação do diabetes mellitus gestacional. [...] (E16)

[...] Com a solicitação de exames especificos, como a glicemia de jejume o teste de tolerância à glicose, a curva glicêmica etc. [...] (E17)

De acordo com as falas não foi citado por nenhum entrevistado, os protocolos de diagnóstico de diabetes mellitus gestacional. A investigação pode ser baseada nos critérios da Organização Mundial da Saúde ${ }^{(11)}$, como também pelo critério da viabilidade financeira e disponibilidade técnica total ou parcial ${ }^{(5)}$.

\section{Categoria 02 - Acompanhamento e encaminhamento}

Os enfermeiros participantes ao identificarem uma gestante com diabetes mellitus gestacional, encaminham estas para o acompanhamento de alto risco na unidade de referência da cidade. $\mathrm{Na}$ rede de atenção à saúde os serviços são planejados de acordo com os critérios de necessidades do usuário e estratificando os riscos visando um atendimento qualificado ${ }^{(12)}$.

[...] gravidez de alto risco é feito o acompanhamento e encaminhamento para o médico. Porque aí o médico irá explicar todos os riscos e fará um acompanhamento e monitoramento mais especializado, de acordo com as alteraçôes encontradas nos exames da gestante. [...] (ES)

Associados à saúde da gestante a assistência de alto risco é essencial reduzir a morbimortalidade infantil-materna, pois a gravidez de alto risco afeta a saúde da mãe e/ou do feto e/ou do recém-nascido, atingindo o seu crescimento e desenvolvimento, ocasionando doenças ao longo da vida ${ }^{(13)}$.

A assistência durante o pré-natal tem a finalidade de identificar problemas, impedindo um resultado desfavorável posteriormente, porém a falha no controle de pré-natal pode fortalecer os riscos materno-infantis contribuindo para a ocorrência de complicações e dificultando a realização de assistências capazes de evitar morbidades grave, morte materna ou fetal ${ }^{(14)}$.

\section{Categoria - Comportamento de risco}

Os hábitos saudáveis, como alimentação e exercícios, foram citados pelos enfermeiros, principalmente quando a gestante é monitorada durante o pré-natal:

[...] A diminuição da ingestão de alimentos ricos em açucares, gera uma ligada ao periodo gestacional, com maior probabilidade de macrossomia, hipoglicemia e prematuridade. [...] (E9)

[...] orientamos quanto a importância de uma alimentação saudável e da introdução de exercícios na rotina da gestante. [...] (E18)

A implementação do consumo de alimentos saudáveis e a introdução de exercícios físicos contribui diretamente com o bem-estar materno melhorando a capacidade aeróbica, auxilia o controle do diabetes tipo II e gestacional, previne o ganho de peso e aumenta a funcionalidade do sistema gastrointestinal. Enfatizando também, a importância da orientação profissional nas escolhas dos exercícios para respeitar os limites físicos das gestantes ${ }^{(15)}$. 


\section{Categoria - "Muitas não podem seguir porque não tem condições financeiras"}

Segundo a Sociedade Brasileira de Diabetes, o tratamento da diabetes gestacional é realizado com base em três modalidades: a educação em saúde, estratégias de automonitorização e ações farmacológicas. Para tanto, a equipe interdisciplinar deve apropriar-se de qualificações necessárias e experiências práticas em atividades de educação em saúde para o êxito na terapêutica ${ }^{(16)}$.

Foi observado em uma das falas o quão é importante considerar as condições de vida da família que tem uma gestante com diabetes. Estudos demostram um déficit na realização das consultas em gestantes com menor nível socioeconômico (17). Dessa forma, o fator socioeconômico está intimamente ligado no êxito do tratamento do diabetes gestacional:
[...] às vezes o nutricionista passa aquela dieta e muitas não podem seguir porque não tem condiçôes financeiras, pois essa alimentação é mais cara e as gestantes não tem condiçôes de faze-las. Eu já tive uma paciente gravida aqui que era diabética e eu dando orientação a ela, me preocupando porque toda consulta que ela vinha o peso aumentava no mês 3 a 4 quilos e eu ia orientando ela sobre a alimentação saudável, né! E ela disse: $E$, tem dias que como um pão porque os vizinhos me dão, e meu marido está desempregado como vou fazer dieta? $E$ aquilo me chocou muito, porque, às vezes, a gente impóe uma coisa e não é a realidade do usuário e a partir desse dia eu já comecei a trabalhar de maneira diferente, conversando, e ouvindo o que ele pode comer [...] (E1)

\section{CONCLUSÃO}

Uma assistência de pré-natal qualificada com estratégias diferenciais na prevenção da diabetes gestacional é decisiva para êxito do controle glicêmico das gestantes, visando sempre o livre acesso aos cuidados de enfermagem oferecidos pelas unidades de saúde da família.

O resultado deste estudo aponta que os enfermeiros possuem o conhecimento adequado sobre o tema. Entretanto, é importante que estes participem de atualizações e capacitações contínuas. Mesmo com as limitações existentes, este estudo proporciona aos profissionais de saúde a compreensão das deficiências na assistência e no acesso dos usuários as unidades de saúde, contribuindo para o não acompanhamento adequado durante o período de pré-natal.

\section{REFERÊNCIAS}

1. Oliveira GZ, Guimarães SZS, Lavado MM. Recém-nascidos grandes para a idade gestacional em gestantes diabéticas do pré-natal de alto risco de Itajaí: fatores de risco. Arq. Catarin Med. 2017; jan-mar; 46(1): 80-96.

2. Consenso "Diabetes Gestacional": Atualização 2017. Revista Portuguesa de Diabetes. 2017; 12(1): 24-38.

3. Bolognani CV, Souza SS, Calderon, IMP. Diabetes mellitus gestacional - enfoque nos novos critérios diagnósticos. Comunicação em Ciências da Saúde, 2011; 22(sup. 1): 31-42.

4. Hod M, Kapur A, Sacks DA, Hadar E, Agarwal M, Di Renzo GC, et al. The International Federation of Gynecology and Obstetrics Initiative on gestational diabetes mellitus: A pragmatic guide for diagnosis, management, and care. Int J Gynaecol Obstet. 2015; 131(Suppl 3): S173-211.

5. Febrasgo. Rastreamento e diagnóstico de diabetes mellitus gestacional no Brasil. Femina 2019; 47(11):786-96.

6. Ministério da saúde (Brasil). Rastreamento e diagnóstico de diabetes mellitus gestacional no Brasil. Organização Pan-Americana da Saúde. Federação Brasileira das Associações de Ginecologia e Obstetrícia. Sociedade Brasileira de Diabetes. Brasília-DF, 2017

7. Associação Brasileira para o Estudo da Obesidade e da Síndrome Metabólica. Diretrizes brasileiras de obesidade. 4.ed. São Paulo, São Paulo. 2016.

8. Ministério da saúde (Brasil). Protocolos da Atenção Básica: Saúde das Mulheres. Instituto Sírio-Libanês de Ensino e Pesquisa. Brasília, 2016.
9. Minayo MCS. O desafio do conhecimento: Pesquisa qualitativa em saúde. São Paulo: Hucitec, 2013.

10. Silva Junior JR, Souza ASR, Agra KF, Cabral Filho JE, Alves JGB. Diabetes mellitus gestacional: importância da produção de conhecimento. Rev. Bras. Saúde Mater. Infant. 2016; 16(2).

11. Zuccolotto DCC, Crivellenti LC, Franco LJ, Sartorelli DS. Padrões alimentares de gestantes, excesso de peso corporal materno e diabetes gestacional. Rev. Saúde Pública. 2019; 53.

12. Mendes FS. Diabetes Mellitus Gestacional: elaboração, adequação cultural e validação de material educativo para o autocuidado de gestantes. Guia da gestante com diabetes gestacional. Belo Horizonte/MG, 2019.

13. Fernandes JA, Campos GWS, Francisco PMSB. Perfil das gestantes de alto risco e a cogestão da decisão sobre a via de parto entre médico e gestante. Saúde debate. 2019; 43(121).

14. Ministério da Saúde (Brasil). Gestação de Alto Risco: Manual Técnico. Brasília, DF. 2012.

15. Padilha PC, Sena AB, Nogueira JL, Araújo RPS, Alves PD, Ac-

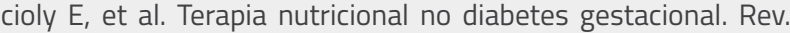
Nutr. 2010; 23(1).

16. Sociedade Brasileira de Diabetes (Brasil). Conduta terapêutica no Diabetes tipo 2: algoritmo da Sociedade Brasileira de Diabetes. São Paulo; 2019; 18.

17. Domingues RMSM, Leal MC, Hartz MAZ, Marcos Augusto Bastos Dias MAB, Vettore MV. Acesso e utilização dos serviços de pré-natal no Sistema Único de Saúde da cidade do Rio de Janeiro, Brasil. Rev. bras. epidemiol. 2013; 16 (04). 